\title{
A Rare Case of Severe Starvation-induced Ketoacidosis in a Patient with Recurrent Pancreatitis
}

\author{
Kok Hoe Chan ${ }^{1}$, Amr Ramahi ${ }^{1}$ \\ 1. Internal Medicine, Saint Michael's Medical Center, Newark, USA
}

Corresponding author: Kok Hoe Chan, kokhoedelcos.chan@gmail.com

\begin{abstract}
Starvation-induced ketoacidosis in non-diabetic and non-pregnant, otherwise healthy patients is not common. In an otherwise normal healthy individual, short-term starving will only result in mild ketosis. Nonetheless, the effects of ketosis can become more severe if there is stress and insulin resistance, such as in pregnant or lactating woman or in very young individual such as neonates. We report a case of severe starvation-induced ketoacidosis in a non-diabetic and non-pregnant 37-year-old African American female patient with a history of multiple recurrent pancreatitis. The patient was initially presented to the emergency department with abdominal pain, nausea and vomiting over two days. The patient also reported starving for two days prior to admission. Biological findings, however, showed a severe degree of metabolic acidosis with an increased anion gap. Serum glucose was normal and 3+ ketonuria were present. Lactic acid was $1.7 \mathrm{mmol} / \mathrm{L}$ with no uremia. Salicylate acid, acetaminophen and ethanol level were normal. The patient's beta-hydroxybutyrate level elevated with ketonuria, suggestive of ketoacidosis as the cause of metabolic acidosis. To our knowledge, the presenting case was novel as no case reports or case series have been reported in these groups of patients. Short-term starvation, if it occurs during periods of stress and medication, may result in life-threatening ketoacidosis, even among non-diabetic women and non-pregnant patients. Awareness of this condition may facilitate prompt recognition and proactive treatment for dietary and stress control.
\end{abstract}

Categories: Gastroenterology, Nephrology

Keywords: starvation, ketoacidosis, recurrent pancreatitis

\section{Introduction}

Ketoacidosis is defined as metabolic acidosis secondary to accumulation of ketone bodies. Diabetic ketoacidosis is the most common cause of ketoacidosis, followed by alcoholic ketoacidosis and rarely starvation ketoacidosis. Starvation-induced ketoacidosis in non-diabetic and non-pregnant, otherwise healthy patients is not common. In a normal healthy individual, short-term starving will only result in mild ketosis. Nonetheless, the effects of ketosis can become more severe if there is a relatively stress and insulin resistance, such as in pregnant or lactating woman or in very young individual such as neonates [1-5]. We report a unique case of severe starvation-induced ketoacidosis in a non-diabetic and non-pregnant patient with a history of multiple recurrent pancreatitis. To our knowledge, the presenting case was novel as no case reports or case series have been reported in these groups of patients.

Received 12/02/2019

gan $12 / 15 / 2019$ Published 03/22/2020

\section{(๑) Copyright 2020}

Chan et al. This is an open access article distributed under the terms of the Creative Commons Attribution License CC-BY 4.0., which permits unrestricted use, distribution, and reproduction in any medium, provided the original author and source are credited.

\section{Case Presentation}

A 37-year-old African American female with a past medical history of controlled asthma (no recent exacerbation or corticosteroid use) and multiple episodes of recurrent pancreatitis that is not related to diabetes (last episode was one year prior to admission) presented to the emergency department with abdominal pain, nausea and vomiting over two days. The patient described the lower abdominal pain as sharp and intermittent in nature, rated as 10/10 in intensity, radiating to the back, with no worsening or relieving factors and associated with multiple bouts of nausea and vomiting. The patient reported starving for two days prior to admission, as she had not been able to take in foods and worried that food would worsen the abdominal pain. Otherwise, the patient denied fever, chills, rigors, changes in bowel movement and urinary symptoms. The patient also denied vaginal discharge, and reported no history of sexually transmitted diseases or oral contraceptives and was not sexually active. The patient's last menstrual period was a few days prior to admission. She also denied any recent sick contact or travel. The patient was not on any medications and supplements such as acetaminophen, salicylates, isoniazid or iron. The patient also denied the use of tobacco, alcohol and illicit drugs.

In the emergency room, the patient started to complain of shortness of breath, tachypnea and acute respiratory distress. Initial vitals showed a blood pressure of 152/104 $\mathrm{mmHg}$, a heart rate of $142 \mathrm{bpm}$, a respiratory rate of $24 \mathrm{bpm}$ and a saturation rate of $98 \%$ on $2 \mathrm{~L} / \mathrm{min}$ of oxygen. Abdominal examination showed soft, non-distended, diffuse tenderness over the lower abdomen, no hepatosplenomegaly and normal bowel sounds. Lung and cardiovascular examinations were otherwise normal. Complete metabolic 


\section{Cureus}

profile showed sodium $132 \mathrm{mmoL} / \mathrm{L}$, potassium $6.4 \mathrm{mmoL} / \mathrm{L}$, bicarb $5 \mathrm{mmoL} / \mathrm{L}$, chloride of $100 \mathrm{mmoL} / \mathrm{L}$, corrected anion gap $29 \mathrm{mmoL} / \mathrm{L}$, lipase was $97 \mathrm{U} / \mathrm{L}$ and $\mathrm{HbA} 1 \mathrm{c} 4.4 \%$. Arterial blood gas showed pH 6.88, $\mathrm{pCO}_{2}$ $12 \mathrm{mmHg}, \mathrm{pO}_{2} 162 \mathrm{mmHg}$ and $\mathrm{HCO}_{3} 2.7 \mathrm{mmHg}$. Delta (delta) gap was 0.9, and the patient was experiencing mixed anion gap metabolic acidosis. Urine anion gap was $31.7 \mathrm{mmol} / \mathrm{L}$, with urine $\mathrm{pH}$ of $5.5,2+$ protein, no glycosuria and no urine casts observed on urine microscopy. Serum glucose was normal (128 mg/dL) and 3+ ketonuria were present. Lactic acid was $1.7 \mathrm{mmol} / \mathrm{L}$ with no uremia (blood urea nitrogen was $6 \mathrm{mg} / \mathrm{dL}$ ). Lipase was $73 \mathrm{U} / \mathrm{L}$ (within normal range). Salicylate acid, acetaminophen and ethanol level were normal. The patient's beta-hydroxybutyrate level was more than 4.5 and 3+ ketonuria, suggestive of ketoacidosis as the cause of metabolic acidosis. The workup for methanol, ethylene glycol, isopropanol and paraldehyde was negative; however, the patient had an elevated osmolar gap, with a gap of $>20$ which was thought to be from the ketoacidosis. Chest X-ray was unremarkable. EKG showed sinus tachycardia (with heart rate of $130 \mathrm{bpm}$ ), normal axis and no ST-T wave abnormalities. CTs of the abdomen and pelvis did not show any signs of acute pancreatitis, pancreatic atrophy or peripancreatic fluids. There was a large uterine fibroid noted at the endometrial cavity. A transvaginal ultrasound showed two discrete fibroids measuring $2.3 \times 1.5 \times 2 \mathrm{~cm}$ and $4.7 \times 2.6 \times 3.4 \mathrm{~cm}$ on the subserosa (Figure 1) and intramural (Figure 2) of the endometrium, respectively. The lower abdominal pain may have been related to the uterine fibroids, as she had negative pregnancy test and no previous gynecological problems.

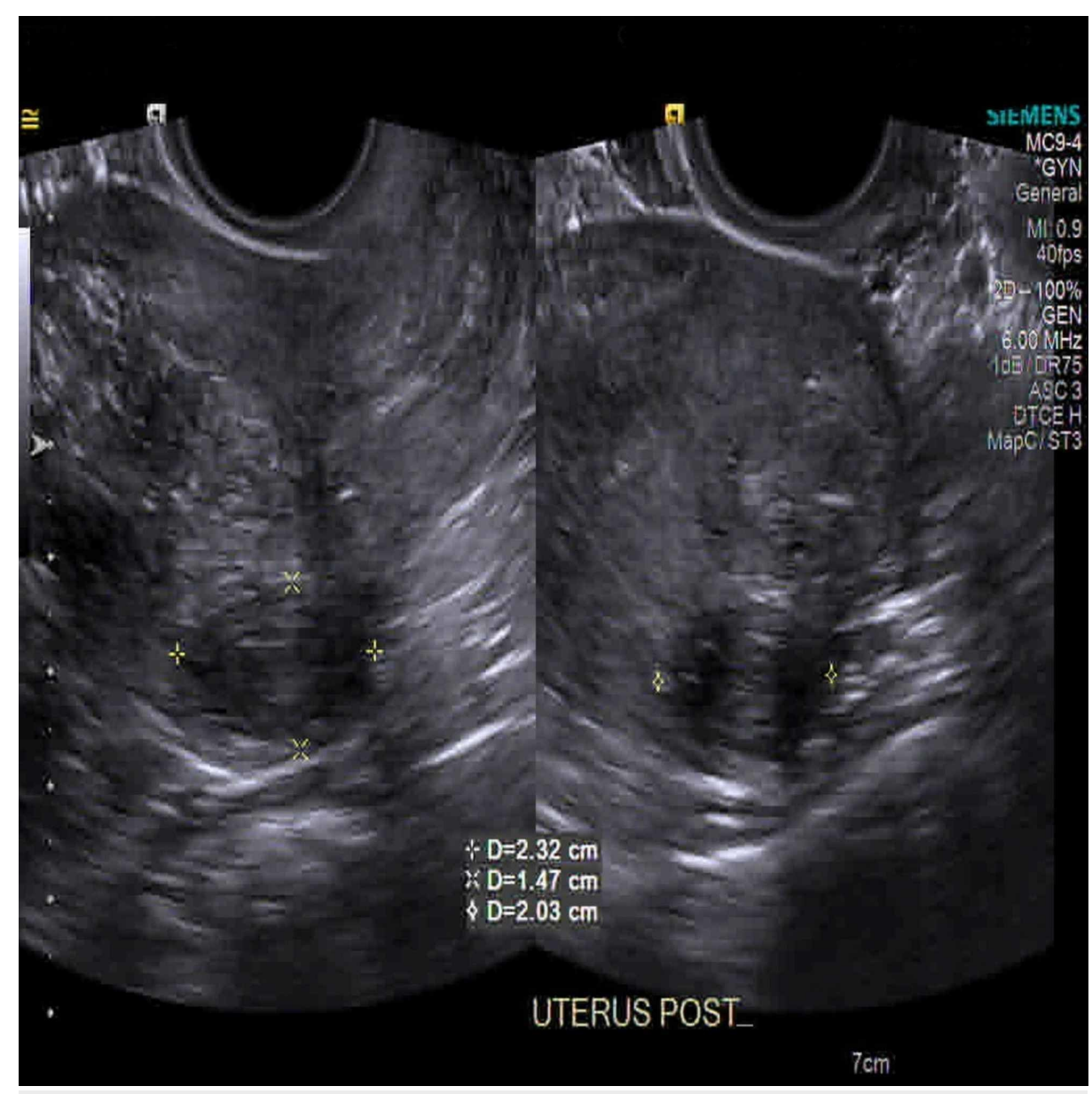

FIGURE 1: Partially subserosal fibroid along the left posterior fundus, measuring $2.3 \times 1.5 \times 2 \mathrm{~cm}$ 


\section{Cureus}

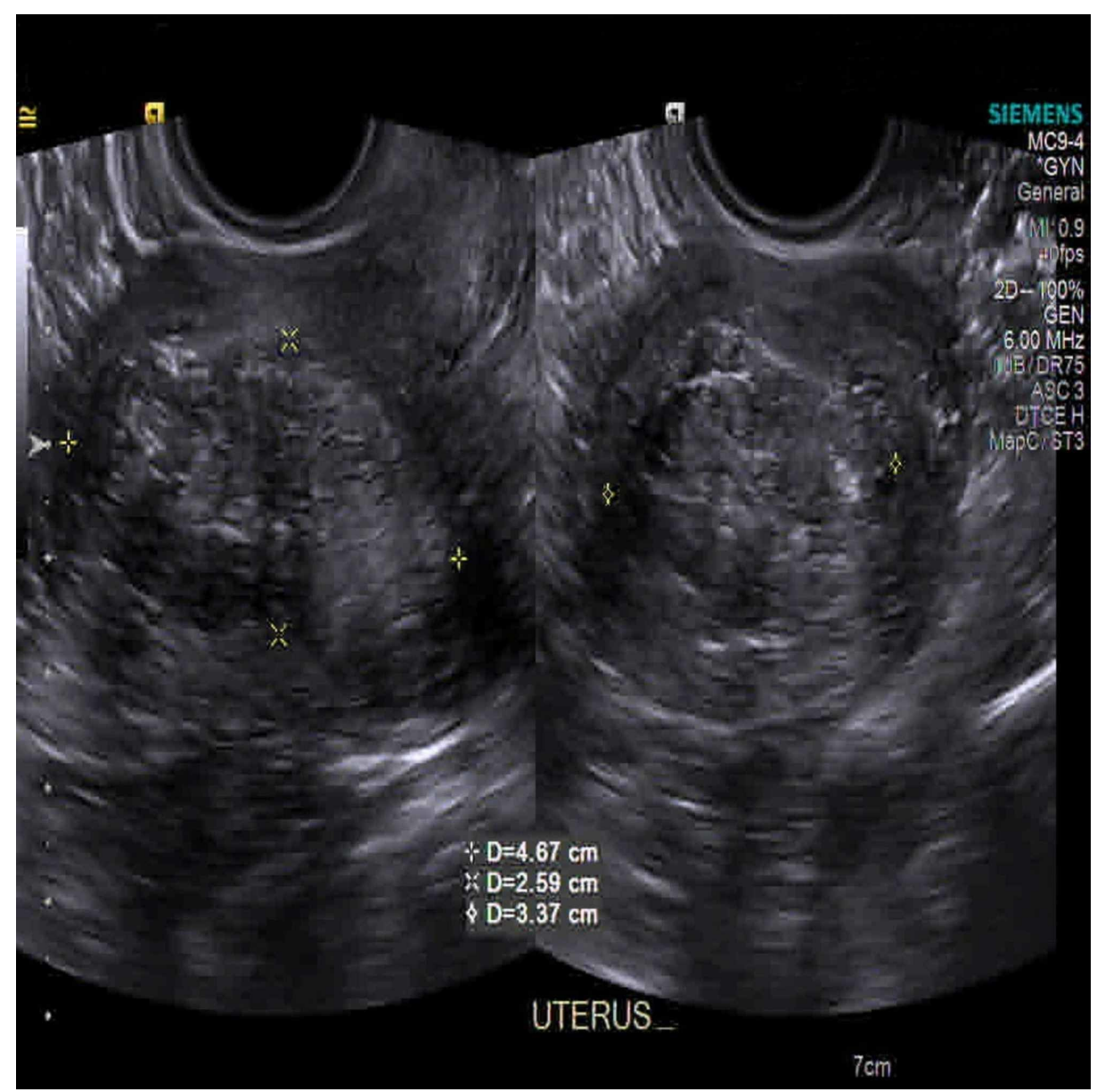

FIGURE 2: Intramural fibroid along the left posterior fundus, measuring $4.7 \times 2.6 \times 3.4 \mathrm{~cm}$

We diagnosed the patient with starvation ketoacidosis due to vomiting, two days of starvation and the absence of other causes of high anion gap metabolic acidosis. Patient was given six ampoules of sodium bicarbonate at the emergency department and was then sent to intensive care unit (ICU). The patient received supplemental oxygen, dextrose and a bicarbonate drip in the ICU. The patient developed refeeding symptoms with hypokalemia, hypomagnesemia and hypophosphatemia. Electrolytes were replenished, and patient's anion gap was improved and resolved with dextrose and fluid infusion.

\section{Discussion}

Ketoacidosis is defined as metabolic acidosis secondary to accumulation of ketone bodies, namely acetone, acetoacetate and beta-hydroxybutyrate. Of these three known ketone bodies, beta-hydroxybutyric acid is a hydroxy acid (as its name implies), acetone is a pure ketone and acetoacetic acid is a true chemical ketoacid. The generation of ketone bodies in the liver is typically stimulated when there is a low insulin/glucagon ratio. Low insulin levels due to either absolute or relative hypoglycemia (catabolic state) will activate hormone-sensitive lipases, leading to the fatty acid generation from lipolysis of adipose tissues and ultimately transported to liver through bloodstream for the production of ketone bodies via beta-oxidation. The hepatic generation of ketone bodies is a normal physiological response to fasting, and it produces an alternative energy source for the brain and other vital organs [1,6-9].

Mild ketosis generally develops after 12 to 14 hours of fasting. If fasting continues, the ketoacid concentration in the plasma will continue to rise, peaking after 20 to 30 days at a concentration of 8 to 10 $\mathrm{mmol} / \mathrm{L}$, at which level the hepatic ketone body synthesis equilibrates with brain and other essential organ tissues' utilization of those ketone bodies, with beta-hydroxybutyrate being the major accumulating ketone body [1.8].

There are at least three stabilization mechanisms inside the body to prevent severe ketoacidosis in patients that are fasting, namely stimulation of insulin release, increased sensitivity of adipose tissue to insulin's inhibitory effect on fatty acid release and direct inhibition of lipolysis by the ketone bodies [10]. Additionally, there will also be an increased rate of central nervous system ketoacid uptake and peripheral 
tissue ketone utilization. These typically decrease the ketoacid in the body and prevent the development of severe ketoacidosis $[11,12]$.

Our case is unique as the patient was otherwise healthy, non-diabetic, non-pregnant and non-lactating but presented with severe starvation ketoacidosis. We postulate that our patient may have had a defect in the breaking mechanisms. Our theory indicates that due to the multiple bouts of acute pancreatitis in the past, the patient's pancreas was unable to release insulin appropriately in response to severe ketoacidosis. It is also interesting to note that the patient developed severe starvation ketoacidosis with just two days of starving. It usually takes much longer for this to happen. In the literature, there are case reports discussing the association of low carbohydrate diet with ketosis. Our patient might have had a low carbohydrate diet lately, along with acute stressful conditions such as vomiting and starving that led to the development of severe starvation-induced ketoacidosis

\section{Conclusions}

Short-term starvation, if it occurs during periods of stress, may result in life-threatening ketoacidosis, even among non-diabetic women and non-pregnant patients. Awareness of this condition may facilitate prompt recognition and proactive treatment for dietary and stress control, especially for malnourished patients; emergent interventions may also improve.

\section{Additional Information}

\section{Disclosures}

Human subjects: Consent was obtained by all participants in this study. Conflicts of interest: In compliance with the ICMJE uniform disclosure form, all authors declare the following: Payment/services info: All authors have declared that no financial support was received from any organization for the submitted work. Financial relationships: All authors have declared that they have no financial relationships at present or within the previous three years with any organizations that might have an interest in the submitted work. Other relationships: All authors have declared that there are no other relationships or activities that could appear to have influenced the submitted work.

\section{References}

1. Cahill GF Jr: Fuel metabolism in starvation. Annu Rev Nutr. 2006, 26:1-22. https://doi.org/10.1146/annurev.nutr.26.061505.111258

2. Rudolf MC, Sherwin RS: Maternal ketosis and its effects on the fetus . Clin Endocrinol Metab. 1983, 12:413. 10.1016/s0300-595x(83)80049-8

3. Mahoney CA: Extreme gestational starvation ketoacidosis: case report and review of pathophysiology . Am J Kidney Dis. 1992, 20:276. https://doi.org/10.1016/S0272-6386(12)80701-3

4. Owen OE, Caprio S, Reichard GA Jr, Mozzoli MA, Boden G, Owen RS: Ketosis of starvation: a revisit and new perspectives. Clin Endocrinol Metab. 1983, 12:359. 10.1016/s0300-595x(83)80046-2

5. Toth HL, Greenbaum LA: Severe acidosis caused by starvation and stress . Am J Kidney Dis. 2003, 42:16. https://doi.org/10.1016/j.ajkd.2003.07.012

6. Rose BD, Post TW: Clinical Physiology of Acid-Base and Electrolyte Disorders, 5th ed . McGraw-Hill, New York; 2001. 801.

7. Foster DW, McGarry JD: The metabolic derangements and treatment of diabetic ketoacidosis . N Engl J Med. 1983, 309:159. DOI: 10.1056/NEJM198307213090307

8. Reichard GA Jr, Owen OE, Haff AC, Paul P, Bortz WM: Ketone-body production and oxidation in fasting obese humans. J Clin Invest. 1974, 53:508. 10.1172/JCI107584

9. Miles JM, Haymond MW, Nissen SL, Gerich JE: Effects of free fatty acid availability, glucagon excess, and insulin deficiency on ketone body production in postabsorptive man. J Clin Invest. 1983, 71:1554. 10.1172/JCI110911

10. Balasse EO, Féry F: Ketone body production and disposal: effects of fasting, diabetes, and exercise . Diabetes Metab Rev. 1989, 5:247. https://doi.org/10.1002/dmr.5610050304

11. Owen OE, Morgan AP, Kemp HG, Sullivan JM, Herrera MG, Cahill GF Jr: Brain metabolism during fasting. J Clin Invest. 1967, 46:1589. https://doi.org/10.1172/JCI105650

12. Owen OE, Reichard GA Jr: Human forearm metabolism during progressive starvation . J Clin Invest. 1971, 50:1536. 10.1172/JCI106639 